\title{
PHOTOLUMINESCENCE SPECTRA OF THE 580-nm CENTER IN IRRADIATED DIAMONDS
}

\author{
A. A. Khomich, ${ }^{\text {a* }}$ R. A. Khmelnitskii, ${ }^{b}$ O. N. Poklonskaya, ${ }^{c}$ \\ A. A. Averin, ${ }^{\text {d }}$ S. N. Bokova-Sirosh, ${ }^{\text {e N. A. Poklonski, }}$ \\ V. G. Ralchenko, ${ }^{e, f}$ and A. V. Khomich ${ }^{\mathrm{a}}$
}

UDC 535.37;548.736.15

The formation mechanisms of the zero-phonon line optical center at $580 \mathrm{~nm}$ (H19 center) in photoluminescence spectra of irradiated natural diamonds and those deposited from the vapor phase were studied after their hightemperature vacuum annealing. The photoluminescence band intensity of the H19 center was shown to increase exponentially as the annealing temperature increased. Temperature dependences of photoluminescence spectra and local mechanical stress effects on the position and full width at half-height of the 580-nm zero-phonon line optical peak led to the conclusion that the H19 optical center was a complex intrinsic vacancy defect.

Keywords: diamond, photoluminescence, zero-phonon line, neutron irradiation, ion implantation, defect.

Introduction. Diamond materials with room-temperature photoactive centers have bright and stable photoluminescence (PL) with high quantum efficiency and narrow lines. This in combination with chemical and radiation resistance and a lack of cytotoxicity makes diamond an attractive material for quantum optics and biomarkers [1,2]. Several new centers giving narrow PL lines in various visible and IR spectral regions were studied in detail because diamond was promising for designing single-photon emitters [3]. Impurities typically have very low equilibrium solubilities and diffusion coefficients in diamond. Therefore, it seemed natural already long ago to use ion implantation as an alternate method for doping diamond. Ion implantation and irradiation by electrons or neutrons followed by thermal annealing are standard procedures for creating most photoactive (optical) centers in diamond [4]. New optical centers are continually sought and their spectral properties are studied for photonics applications despite the fact that several hundred optical centers are already known in diamond [5].

A band with a zero-phonon line (ZPL) at $580 \mathrm{~nm}(2.137 \mathrm{eV})$ and distinct vibronic structure on the phonon tail that were not previously observed in diamond PL spectra were detected in PL spectra of natural diamond and those from chemical vapor deposition (CVD) implanted with D-ions and vacuum annealed at $1000-1650^{\circ} \mathrm{C}$ (for $1 \mathrm{~h}$ at each temperature) [6]. This same band with a ZPL at $580 \mathrm{~nm}$ was observed in PL spectra of natural and CVD diamonds implanted with H-ions [7] or irradiated with fast reactor neutrons [8] after high-temperature annealing. It is noteworthy that this band did not appear in the optical absorption spectrum. It strengthened smoothly as the annealing temperature was increased. The annealing parameters did not correlate with the $\mathrm{N}$ and Si concentration and speciation in diamond or with the contents of starting or implanted H/D. A classification for the optical centers in diamond, including radiation-induced ones, was proposed [9]. In particular, centers formed by heat treatment after irradiation were designated $\mathrm{H}$ (heat treatment), from $\mathrm{H} 1$ to $\mathrm{H} 18$ [10]. The optical center at $580 \mathrm{~nm}$ with a ZPL was called H19 according to this classification [11].

A band of similar spectral position and shape was observed in electroluminescence spectra of diamond irradiated by a focused beam of $\mathrm{C}^{3+}$ ions ( $6 \mathrm{MeV}$ kinetic energy) and annealed at $1000^{\circ} \mathrm{C}$ [12], where it was erroneously interpreted

* To whom correspondence should be addressed.

${ }^{\mathrm{a}}$ V. A. Kotelnikov Institute of Radio Engineering and Electronics, Fryazino Branch, Russian Academy of Sciences, 1 Vvedenskogo Sq., Fryazino, 141190, Moscow Region, Russia; email: antares-610@yandex.ru; ${ }^{b}$ P. N. Lebedev Physical Institute, Russian Academy of Sciences, Moscow, 117924, Russia; 'Belarusian State University, Minsk, 220030, Belarus; email: poklonski@bsu.by; ${ }^{\mathrm{d}}$ A. N. Frumkin Institute of Physical Chemistry and Electrochemistry, Russian Academy of Sciences, Moscow, 119071, Russia; 'A. M. Prokhorov General Physics Institute, Russian Academy of Sciences, Moscow, 117942, Russia; ${ }^{\mathrm{f}}$ Harbin Institute of Technology, Harbin, 150001, China. Translated from Zhurnal Prikladnoi Spektroskopii, Vol. 86, No. 4, pp. 539-548, July-August, 2019. Original article submitted April 22, 2019. 
as a mixed band of an $\mathrm{N} V$-center that is usually found at $575 \mathrm{~nm}$. The high sensitivity of the band intensity at $580 \mathrm{~nm}$ to the applied electric field could be useful for applications as sensors [12]. Later, researchers hypothesized that the band with a ZPL (580 nm) in PL and electroluminescence spectra [13] was due to the center observed previously [14, 15]. Several optical centers with a ZPL, most of which are still unidentified, were detected in those studies in PL spectra of diamond after room-temperature irradiation with electrons [electron kinetic energy $300 \mathrm{keV}$, dose (1-20) $10^{19} \mathrm{~cm}^{-2}$ ]. An optical center with a ZPL at $580 \mathrm{~nm}$ was observed among these optical centers. However, its phonon structure was completely different. The center itself was interstitial in nature and fully annealed already at $500^{\circ} \mathrm{C}[14,15]$.

The present work studied the conditions and formation mechanisms of H19 centers (ZPL at $580 \mathrm{~nm}$ ) in natural and CVD diamonds resulting from irradiation followed by high-temperature annealing. The temperature dependences of the parameters and the impact of mechanical stresses on the PL band associated with this center were studied.

Experimental. Polished plates of natural diamond single crystals of types IIa and IaA and polycrystalline CVD diamonds $\sim 500 \mu \mathrm{m}$ thick that were grown in an RF plasma in $\mathrm{H}_{2}-\mathrm{CH}_{4}$ mixtures were studied. The concentration of solitary $\mathrm{N}$ substituted into CVD diamond was determined by the literature method [16] as $10^{17}-3 \cdot 10^{18} \mathrm{~cm}^{-3}$. Implantation of $\mathrm{D}^{+}$and $\mathrm{H}^{+}$ions used energies $<350 \mathrm{keV}$ on a High Voltage Engineering Europe ion accelerator. Six implantation areas $\left(0.7 \times 1.5 \mathrm{~mm}\right.$, dose from $2 \cdot 10^{16}$ to $\left.1.2 \cdot 10^{17} \mathrm{~cm}^{-2}\right)$ separated by areas of undamaged diamond were formed on each plate of natural and CVD diamond. Another group of natural and CVD diamonds was irradiated in the water channel of an IVV-2M nuclear reactor in a fast neutron beam of $\sim 10^{14} \mathrm{~cm}^{-2} \cdot \mathrm{s}^{-1}$ (neutron kinetic energy $>0.1 \mathrm{MeV}$, flux from $2 \cdot 10^{19}$ to $2 \cdot 10^{20} \mathrm{~cm}^{-2}$ ) [17]. Implanted (irradiated) samples were vacuum annealed in a graphite furnace (residual gas pressure $1 \mathrm{mPa}$ ) sequentially at $T_{\mathrm{a}}=200-1650^{\circ} \mathrm{C}$ (60 min at fixed temperature). Samples were etched with hot $\mathrm{H}_{2} \mathrm{SO}_{4}+\mathrm{K}_{2} \mathrm{Cr}_{2} \mathrm{O}_{7}$ solution at $\sim 180^{\circ} \mathrm{C}$ to remove the thin surface layer of graphite formed after high-temperature vacuum annealing. PL and Raman spectra were measured on LABRAM HR800, Nanofinder HE, Bruker Senterra Raman, and Renishaw's in Via Reflex spectrometers with laser excitation at $\lambda_{\mathrm{ex}}=473,488$, and $532 \mathrm{~nm}$ at room temperature $(300 \mathrm{~K})$. Separate measurements were made at $80 \mathrm{~K}$ and in the range $300 \leq T \leq 800 \mathrm{~K}$. A Linkam TS1500 temperature accessory was used to measure PL spectra at elevated temperatures.

Results and Discussion. $P L$ spectra with annealing at $T_{a}>1600^{\circ} \mathrm{C}$. Figure la shows PL spectra of natural and synthetic diamonds irradiated by fast neutrons or implanted with hydrogen isotopes after annealing at $T_{\mathrm{a}} \geq 1630^{\circ} \mathrm{C}$. All spectra showed a doublet at 580 and $598 \mathrm{~nm}$ and a structured phonon tail, the peak positions of which were insensitive to the method of diamond radiation damage and the impurity content. Extensive radiation damage and high-temperature annealing were necessary conditions for forming $\mathrm{H} 19$ centers (ZPL $580 \mathrm{~nm}$ ) in the diamonds. The short-wavelength part of the spectrum had a diamond Raman band $\left(v_{R}=1332 \mathrm{~cm}^{-1}\right)$ and an $\mathrm{H} 3$ center PL band (band of phonon repetitions with a maximum near $525 \mathrm{~nm}$; two $\mathrm{N}$ atoms separated by a $2 \mathrm{~N} V$ vacancy [8]). The intensity of the $\mathrm{H} 3$ band was determined by the number of 2N $V$ centers and the PL spectra excitation wavelength (Fig. 1a). The excitation efficiency of the 580-nm center (H19) increased by $\sim 3$ times on going from $\lambda_{\mathrm{ex}}=473$ to $488 \mathrm{~nm}$ according to the intensity ratio of the PL bands and diamond $I_{\mathrm{R}}$ (Dia) peak in Raman spectra; on going from 488 to $532 \mathrm{~nm}$, by another $\sim 2.5$ times. This was characteristic of the PL excitation spectrum of this center.

Other bands besides the doublet at 580 and $598 \mathrm{~nm}$ and the structured phonon tail were recorded in PL spectra of irradiated diamonds. Thus, the CVD diamond film was enriched in Si atoms because it was deposited onto a Si substrate. Therefore, all PL spectra of CVD diamonds contained a band near $738 \mathrm{~nm}$ for a Si vacancy (SiV) [10, 18]. The PL spectrum taken from the fine-grained nucleated (substrate) side of a CVD diamond plate had an $\mathrm{Si} V$ band that was 2-3 orders of magnitude greater than that from the growth side because Si migrated from the substrate to the plasma only in the early growth stage [19]. Spectra of diamonds implanted with hydrogen isotopes (Fig. 1a, spectra 2 and 3) contained sets of narrow red bands at 720-780 $\mathrm{nm}$ with varying intensities along the implantation area [6] and a narrow strong band at $511 \mathrm{~nm}$ that is usually observed for pure type IIa diamond after implantation of hydrogen or carbon ions and annealing at $T_{\mathrm{a}}>1000^{\circ} \mathrm{C}$ [20]. The description of these is beyond the scope of the present work. A continuous graphitized layer formed in the area with the most radiation damage for diamond implanted with hydrogen isotopes at doses $>8 \cdot 10^{16} \mathrm{~cm}^{-2}$ followed by high-temperature annealing [21]. As a result, PL spectra 2 and 3 (Fig. 1a) exhibited interference with a period corresponding to the depth of this layer $(\sim 1.9 \mu \mathrm{m})$.

Photochromism is characteristic of many optical centers in diamond [22, 23]. Spectra of diamonds irradiated by neutrons and annealed at $T_{\mathrm{a}}=1680^{\circ} \mathrm{C}$ with strong PL lines at $580 \mathrm{~nm}$ changed shape at excitation beam $\left(\lambda_{\mathrm{ex}}=532 \mathrm{~nm}\right)$ powers from 0.1 to $20 \mathrm{~mW}$. Also, several Raman bands characteristic of natural diamond and CVD diamond with extensive radiation damage were recorded in the short-wavelength part of the spectrum [17]. Their spectral positions changed in concert with the excitation wavelength (Fig. 1b, range $490-525 \mathrm{~nm}$ ). 

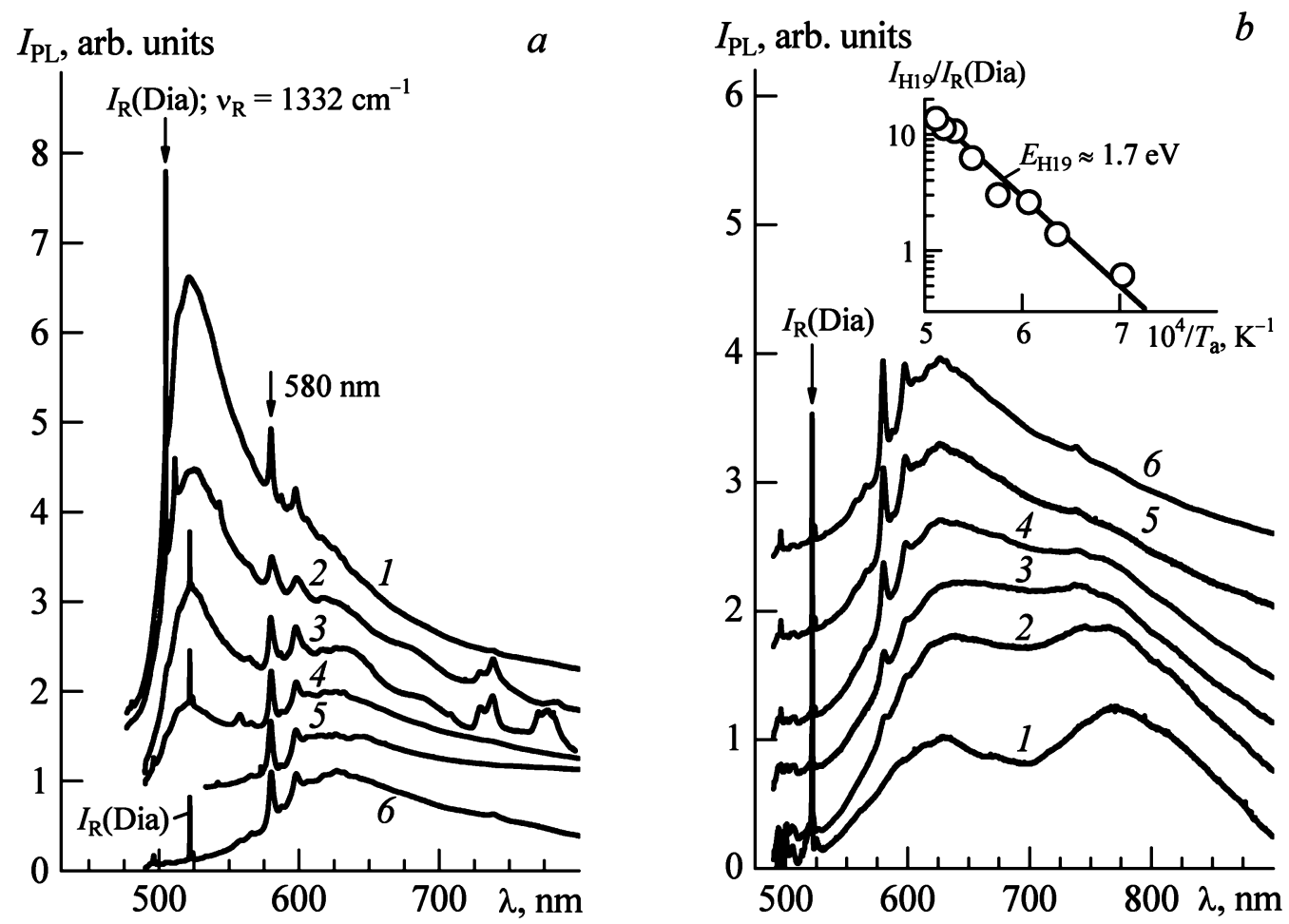

Fig. 1. Photoluminescence spectra of natural diamond irradiated by neutron flux $1 \cdot 10^{20} \mathrm{~cm}^{-2}$ and annealed at $1630^{\circ} \mathrm{C}$ for $1 \mathrm{~h} \mathrm{(1)}$ and at $1665^{\circ} \mathrm{C} \mathrm{(5)} \mathrm{(a)} \mathrm{and} \mathrm{CVD-diamonds}$ [H-ion implantation flux $8 \cdot 10^{16} \mathrm{~cm}^{-2}$ and annealing at $1660^{\circ} \mathrm{C} \mathrm{(2);} \mathrm{D-ion} \mathrm{implantation} \mathrm{flux}$ $1.2 \cdot 10^{17} \mathrm{~cm}^{-2}$ after annealing at $1660^{\circ} \mathrm{C}(3)$; irradiated by neutron flux $2 \cdot 10^{19} \mathrm{~cm}^{-2}$ and annealed at $1680^{\circ} \mathrm{C} \mathrm{(4);} \mathrm{irradiated} \mathrm{by} \mathrm{neutron} \mathrm{flux} 2 \cdot 10^{20} \mathrm{~cm}^{-2}$ and annealed at $1680^{\circ} \mathrm{C} \mathrm{(6)];}$ $\lambda_{\text {ex }}=473(1,2), 488(3,4,6)$, and $532 \mathrm{~nm}(5)$ (a) and CVD-diamond irradiated by neutron flux $2 \cdot 10^{20} \mathrm{~cm}^{-2}$ and annealed at $T_{\mathrm{a}}=1080$ (1), 1300 (2), 1465 (3), 1550 (4), 1610 (5), and $1680^{\circ} \mathrm{C}(6) ; \lambda_{\text {ex }}=488 \mathrm{~nm}$ from the growth side at room temperature; in the inset, semilogarithmic plot of $\mathrm{H} 19$ center integrated intensity $I_{\mathrm{H} 19}$ (ZPL $580 \mathrm{~nm}$ ) normalized to the integrated intensity of diamond Raman line $I_{R}(\mathrm{Dia})$ vs. inverse annealing temperature.

Impact of annealing temperature on PL spectra. Figure $1 \mathrm{~b}$ shows the impact of annealing temperature on the PL spectrum of diamond irradiated by fast neutrons. The band with a ZPL at $580 \mathrm{~nm}$ appeared in spectra of radiationdamaged diamond after annealing at $T_{\mathrm{a}}=1250-1300^{\circ} \mathrm{C}$ (Fig. 1b). A broad band $B$ with a maximum near $770 \mathrm{~nm}$ dominated in PL spectra of irradiated diamond at lower annealing temperatures [20]. The annealing temperature $T_{\mathrm{a}}$ was the main factor affecting the band intensity and width. The full width at half-height $(\Gamma)$ of the $\mathrm{H} 19$ center decreased from 7-8 to 2-3 nm and its intensity increased rapidly as $T_{\mathrm{a}}$ increased (Fig. 1b). PL spectra also recorded a band near $738 \mathrm{~nm}$ for an $\operatorname{Si} V$ center $[10,18]$ and bands with maxima at $\sim 558$ and $566 \mathrm{~nm}$ (Fig. 1a).

The activation energy $E_{\mathrm{H} 19}$ for the increase of integrated intensity $I_{\mathrm{H} 19}$ of an $\mathrm{H} 19$ center (ZPL at $\left.580 \mathrm{~nm}\right)$ of CVD diamond irradiated by neutron fluxes $2 \cdot 10^{19}$ and $2 \cdot 10^{20} \mathrm{~cm}^{-2}$ relative to that of the diamond peak $I_{\mathrm{R}}($ Dia) in Raman spectra as a function of annealing temperature $T_{\mathrm{a}}$ was $E_{\mathrm{H} 19}=1.7 \pm 0.1 \mathrm{eV}$ (Fig. 1b, inset). It should be recalled that the intensity $I_{\mathrm{R}}(\mathrm{Dia})$ of the diamond Raman band itself decreased during annealing of radiation-damaged diamond, i.e., the actual activation energy for the intensity increase of the PL band at $580 \mathrm{~nm}$ (H19) was slightly greater. This quantity was comparable to the activation energy for vacancy migration in diamond ( $2.12 \mathrm{eV}$ for diamond irradiated by high-energy ions [24]; up to $2.3 \mathrm{eV}$ for migration of a neutrally charged vacancy in perfect natural diamonds of types Ia, Ib, and IIa [25]).

Impact of stresses in diamond on PL of the H19 center. Splitting of the ZPL at $580 \mathrm{~nm}$ into at least two components was observed in several PL spectra of a polycrystalline CVD diamond implanted with D-ions (doses from $8 \cdot 10^{16}$ to $12 \cdot 10^{16} \mathrm{~cm}^{-2}$ ) and annealed at $1650-1680^{\circ} \mathrm{C}$ (Fig. 2a). Confocal measurements (exciting laser diameter $\sim 1 \mu \mathrm{m}$ ) showed that this splitting 


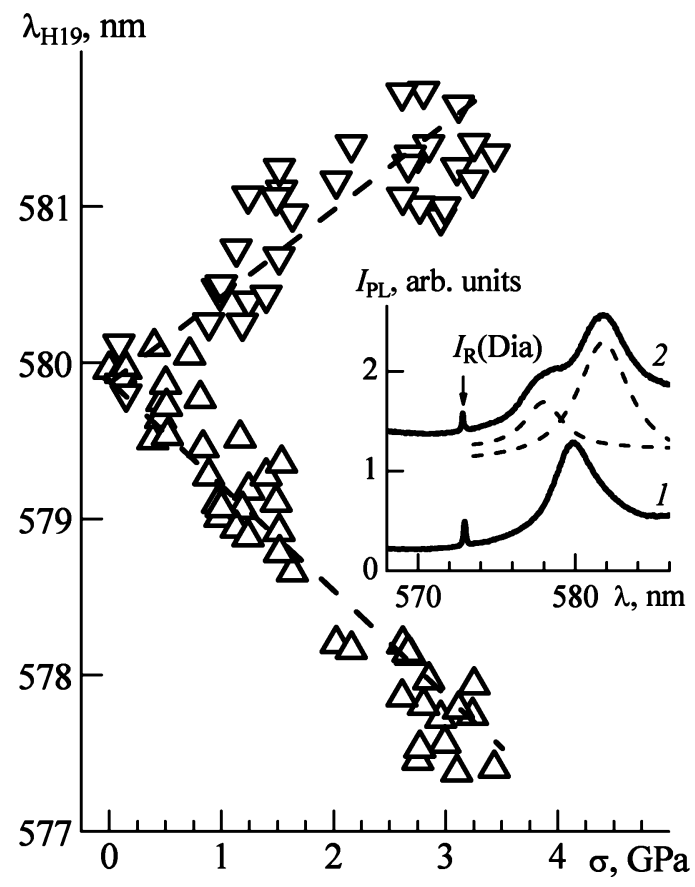

Fig. 2. Dependence of positions of maxima for split ZPL H19 center components in CVD-diamond implanted with D-ions at a dose of $8 \cdot 10^{16} \mathrm{~cm}^{-2}$ and vacuum annealed at $T_{\mathrm{a}}=1640^{\circ} \mathrm{C}$ on local mechanical stress $\sigma$ determined from the splitting of the diamond Raman peak; in the inset, PL spectra at sample points with the minimal (1) and maximal splitting (2) of the H19 center ZPL; dashed lines are two deconvoluted ZPL components; $\lambda_{\text {ex }}=532 \mathrm{~nm}$ from the sample growth side.

was most evident in individual CVD crystallites near intercrystallite boundaries. The diamond peak in Raman spectra split and shifted simultaneously, indicating that the sample had high local mechanical stresses. Local stresses determined using the literature method [26] varied strongly through the sample and reached $\sigma \approx 3.5 \mathrm{GPa}$ at separate points. Figure 2 shows a plot based on $>40$ measurements in various surface sections of a CVD diamond plate. It is noteworthy that intrinsic stresses typically vary over space in polycrystalline diamond films, even untreated ones, and can reach $\sim 9 \mathrm{GPa}$ by concentrating near grain boundaries [27]. Graphitization of intercrystallite boundaries in CVD diamonds during high-temperature annealing could be yet another source of stress $[28,29]$. Annealing polycrystalline CVD diamonds at $T_{\mathrm{a}} \geq 1300^{\circ} \mathrm{C}$ is known to break $\mathrm{C}-\mathrm{H}$ bonds and transform defect diamond into amorphous carbon and turbostratic graphite [28]. This is associated with a sharp increase of the light absorption coefficient [28,29] while retaining the same thermal conductivity [19] and elastic properties [29] of annealed CVD diamond. Graphitization was greatest near intercrystallite boundaries where bound hydrogen was concentrated in CVD diamonds. In turn, high local mechanical stress fields formed in annealed CVD diamonds because diamond and graphite have different specific densities. Radiation damage increased the temperature at which graphitization of the CVD intercrystallite boundaries started because hydrogen atoms redistributed throughout the whole sample [30].

Figure 2 (inset) shows the deconvoluted PL band of the H19 center as two Lorentzian components with, as a rule, a stronger long-wavelength component. The short-wavelength component shifted by up to $2.5 \mathrm{~nm}(9 \mathrm{meV})$. The diamond peak (near $573 \mathrm{~nm}$ ) in the Raman spectrum was noticeably weakened relative to the ZPL of the H19 center for sample sections with high mechanical stresses. The positions of the maxima from the split PL maximum of the H19 center were approximated well by linear functions of the local mechanical stress determined from Raman spectra.

Impact of measurement temperatures on $H 19$ center PL. The ZPL at $580 \mathrm{~nm}$ of the $\mathrm{H} 19$ center shifted to longer wavelengths and broadened as the temperature increased. The low-temperature position of the H19 center ZPL was determined from PL spectra at $80 \mathrm{~K}$. According to the classical model of impurity centers in a solid [31], the shift $\Delta E_{\mathrm{s}}(T)$ of the ZPL maximum with energy $E_{\mathrm{H} 19}(T)$ due to the increased lattice constant and electron-phonon interaction (EPI) caused by heating diamond is proportional to $T^{4}$, where $T$ is the absolute temperature. The expression $\Delta E_{\mathrm{s}}(T)=E_{\mathrm{H} 19}(80)-E_{\mathrm{H} 19}(T)=a T^{2}+b T^{4}$, 

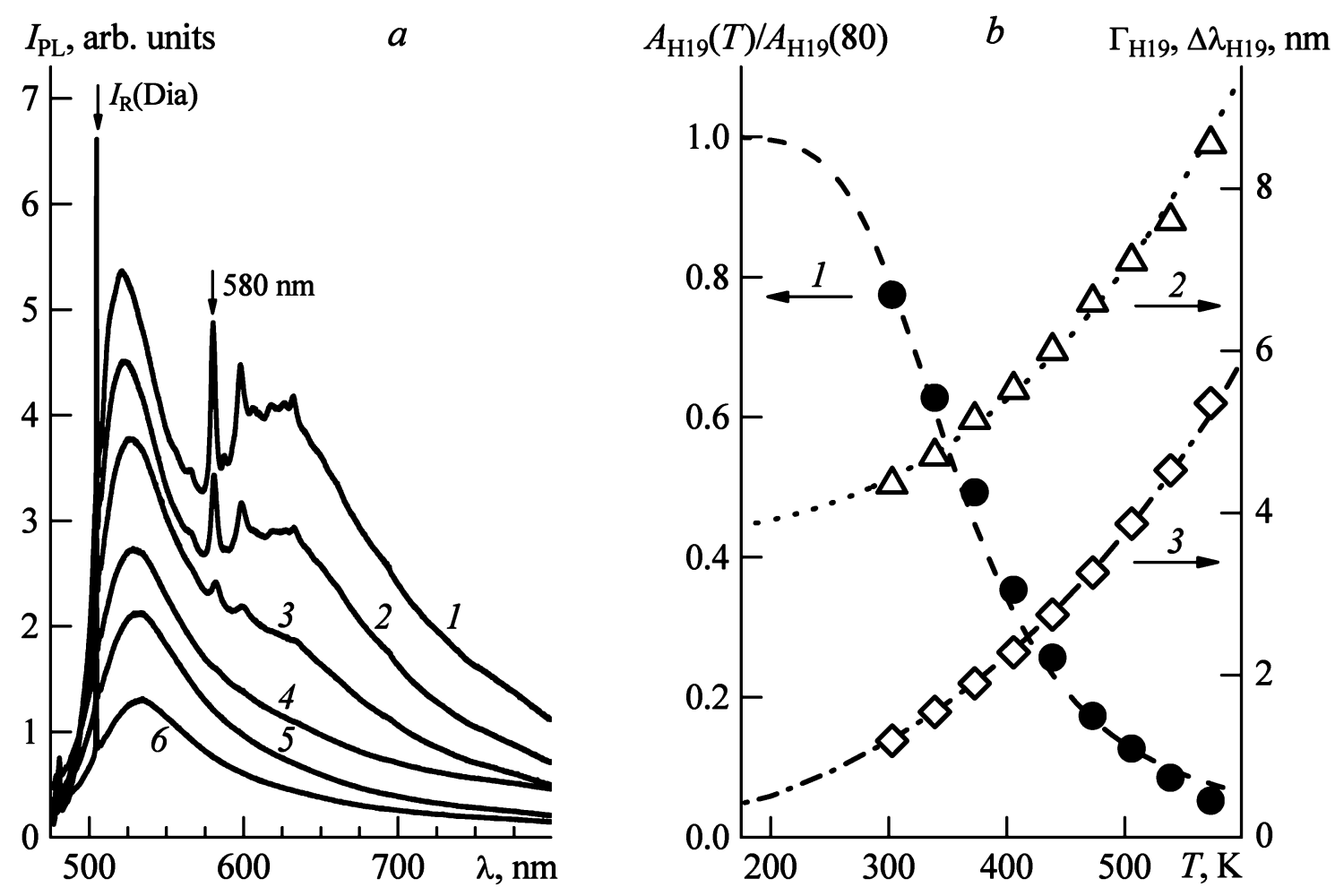

Fig. 3. PL spectra of CVD-diamond irradiated by neutron flux $2 \cdot 10^{19} \mathrm{~cm}^{-2}$ and annealed at $T_{\mathrm{a}}=1700^{\circ} \mathrm{C}$ recorded at $T=20$ (1), 100 (2), 200 (3), 300 (4), 400 (5), and $500^{\circ} \mathrm{C}$ (6); $\lambda_{\mathrm{ex}}=473 \mathrm{~nm}$ from the growth side (a) and temperature dependences of the ratio of integrated intensities of the $\mathrm{H} 19$ center ZPL at temperature $T$ and at $80 \mathrm{~K}$ normalized to the integrated intensity of diamond line $I_{\mathrm{R}}(\mathrm{Dia})$ in Raman spectra $\left[A_{\mathrm{H} 19}(T) / A_{\mathrm{H} 19}(80)\right]$ at the corresponding temperatures (1), full widths at half-height $\Gamma_{\mathrm{H} 19}(2)$, and shifts $\Delta \lambda_{\mathrm{H} 19}(3) ; \lambda_{\mathrm{H} 19}(80)=579 \mathrm{~nm}$.

which also considers softening of chemical bonds in the center excited electronic state, must be used to describe the functions in Fig. 3 [32]. The best agreement for the spectra in Fig. 3, for which the ZPL maximum shifted by 15 meV in the range $30-300^{\circ} \mathrm{C}$, was obtained for $a=4.5 \cdot 10^{-8} \mathrm{eV} / \mathrm{K}^{2}$ and $b=4 \cdot 10^{-14} \mathrm{eV} / \mathrm{K}^{4}$ (Fig. 3b). The quantity $\Delta E_{\mathrm{s}}$ for the $\mathrm{H} 19$ center was half that for an $\mathrm{N}^{-}$center [33]. The temperature dependence of the full width at half-height $\Gamma_{\mathrm{H} 19}$ for the $\mathrm{H} 19$ center obeyed the expression $\Gamma_{\mathrm{H} 19}(\mathrm{~nm})=3.7+2.65 \cdot 10^{-8} T^{3}$ (Fig. $3 \mathrm{~b}$ ). The ratio of integrated intensities $A_{\mathrm{H} 19}(T)$ of the H19 center in the PL spectrum $I_{\mathrm{H} 19}(T)$ and diamond line $I_{\mathrm{R}}(\mathrm{Dia} ; T)$ in the Raman spectrum at temperature $T$ was defined as $A_{\mathrm{H} 19}(T) / A_{\mathrm{H} 19}(80)$ $=\left[1+850 \exp \left(-E_{\mathrm{dp}} / k_{\mathrm{B}} T\right)\right]^{-1}$, where $A_{\mathrm{H} 19}(80)=I_{\mathrm{H} 19}(80) / I_{\mathrm{R}}(\mathrm{Dia} ; 80)=7.5$ is the ratio of the $\mathrm{H} 19$ center ZPL integrated intensity in the PL spectrum to that of the diamond line in the Raman spectrum at $80 \mathrm{~K} ; E_{\mathrm{dp}}$, activation energy for thermal quenching of the ZPL center; and $k_{\mathrm{B}}$, Boltzmann's constant. The activation energy for thermal quenching of the integrated intensity of the $\mathrm{H} 19$ center ZPL was $E_{\mathrm{dp}}=210 \mathrm{meV}$ for CVD diamond irradiated by neutron flux $2 \cdot 10^{19} \mathrm{~cm}^{-2}$ and annealed at $T_{\mathrm{a}}=1700^{\circ} \mathrm{C}$ (Fig. 3b).

Vacancy defects in covalent crystals loosen the lattice. Conversely, interstitial defects strengthen the force constants of vibrational modes [10]. Therefore, large EPI is observed at vacancy defects and small EPI at interstitial ones. Thus, vacancy defects have larger deformation softness $S_{\mathrm{p}}=\left(\hbar \omega_{0}\right)^{-1} \Delta\left(\hbar \omega_{0}\right) / \Delta P$ and thermal softness $S_{\mathrm{t}}=\left(\hbar \omega_{0}\right)^{-1} \Delta \Gamma(T) / \Delta T$, where $\hbar \omega_{0}$ and $\Gamma$ are the ZPL maximum energy and full width at half-height; $\Delta\left(\hbar \omega_{0}\right)$, the shift or splitting of the ZPL by uniaxial pressure $\Delta P$; and $\Delta \Gamma(T)$, change of $\Gamma$ with temperature. The quantities $S_{\mathrm{p}}$ and $S_{\mathrm{t}}$ of the optical center must be compared with those for the ideal crystal lattice. They can be estimated from the dependences of the band gap (diamond energy gap) on temperature and pressure and were $2 \cdot 10^{-12} \mathrm{eV} / \mathrm{Pa}$ and $-5.4 \cdot 10^{-5} \mathrm{eV} / \mathrm{K}$ according to the literature [34]. Hence, $S_{\mathrm{p}}$ and $S_{\mathrm{t}}$ for the ideal diamond crystal structure are $0.36 \cdot 10^{-12} \mathrm{~Pa}^{-1}$ and $1 \cdot 10^{-5} \mathrm{~K}^{-1}$. The values were $S_{\mathrm{p}}=2.2 \cdot 10^{-12} \mathrm{~Pa}^{-1}$ and $S_{\mathrm{t}}=2.1 \cdot 10^{-5} \mathrm{~K}^{-1}$ for the data shown in Figs. 2 and 3. The high $S_{\mathrm{p}}$ and $S_{\mathrm{t}}$ values allow the H19 center to be considered a soft vacancy defect. 


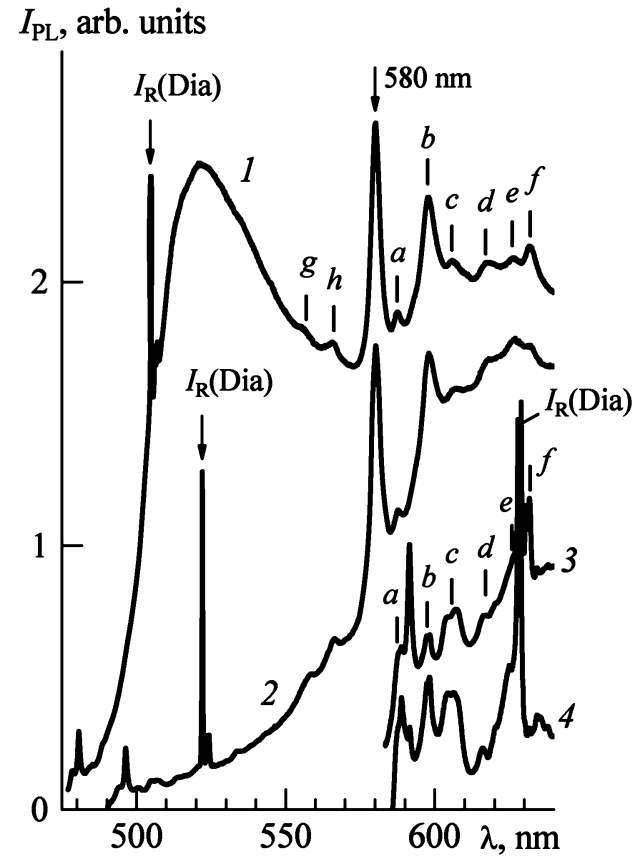

Fig. 4. Photoluminescence spectra of CVD-diamonds irradiated by neutron fluxes $2 \cdot 10^{19}$ (1) and $2 \cdot 10^{20} \mathrm{~cm}^{-2}(2)$ and annealed at $1680^{\circ} \mathrm{C}$; Raman spectra of CVD-diamond irradiated by neutron flux $2 \cdot 10^{19} \mathrm{~cm}^{-2}$ and annealed at $1375(3)$ and $1555^{\circ} \mathrm{C}(4)$; the starting point of spectra 3 and 4 was the position of the $\mathrm{H} 19$ center ZPL in spectra 1 and $2 ; \lambda_{\text {ex }}=473$ (1) and $488 \mathrm{~nm}(2-4)$.

Features of the H19 center phonon tail. One method of studying optical centers and crystal-lattice dynamics uses the phonon tail structure from the corresponding ZPL in absorption and PL spectra. Differences between the ZPL spectral position and features on the phonon tail correspond to either vibrational frequencies at Brillouin-zone critical points or socalled local modes of impurity atoms forming the center [35]. Figure 4 shows PL spectra of CVD diamonds irradiated by fast-neutron fluxes $2 \cdot 10^{19}$ and $2 \cdot 10^{20} \mathrm{~cm}^{-2}$ for $\lambda_{\mathrm{ex}}=473$ and $488 \mathrm{~nm}$.

Features with shifts of 27.5 (a), 63.5 (b), 92 (c), 126 (d), 157 (e), and $174 \mathrm{meV}$ (f) were observed on the H19 center phonon tail. These features were clearly resolved in spectra of the studied natural and CVD diamonds after high-temperature annealing and rapid growth of H19 center PL intensity (Fig. 1b). Their relative intensities were practically independent of the PL excitation wavelength and the diamond impurity profile, in particular, on the intensity of the $\mathrm{H} 3$ center phonon repetition band with a maximum near $520 \mathrm{~nm}$ and a PL band with a maximum at $738 \mathrm{~nm}$ from a Si $V$ center (Figs. 1a and 4). The strongest band $b$ on the H19 center phonon tail was shifted relative to the ZPL by $63.5 \mathrm{meV}$ whereas the minimal phonon frequency in diamond at a Brillouin-zone special point (TA-phonon at point $L,\langle 111\rangle$ ) is $71 \mathrm{meV}$ [10]. The spectral positions of features $d$ and $e$ in PL spectra of the H19 center (Fig. 4) agreed well with those in spectra of diamond density of phonon states $[10,36]$, which was not valid for several other features designated in Fig. 4 . The features on the center phonon tail cannot be attributed to local vibrational modes of impurity atoms because the impurity profile of radiation damaged diamond was not related to the shape and strength of the H19 center PL band [35]. PL spectra were also missing a local mode at 38-40 meV that was characteristic of diamond centers with a solitary vacancy in their structure [10] (Figs. 1, 2, and 4).

A comparison of Raman and PL spectra showed that all features on the H19 center phonon tail correlated with Raman maxima (Fig. 4b, spectra 3 and 4) at 220, 260, 335, 500, 528, 693, 755, 1009, 1237, 1385, and $1412 \mathrm{~cm}^{-1}(27.5,32.1,48.3,62$, $65.3,85.9,93.6,124.7,139,171.6$, and $175 \mathrm{meV}$, respectively). This set of bands in diamond Raman spectra was proposed to result from high levels of radiation damage. High-temperature annealing restored only partially the diamond crystal structure $[6,11]$. Amorphized regions in which the phonon correlation length was $\sim 1 \mathrm{~nm}$, i.e., only three diamond-lattice lengths, the cubic modification could not be fully restored. Regions with high linear defect concentrations [37] or (under the influence of powerful laser radiation) other noncubic modifications of $s p^{3}-\mathrm{C}$ were formed [38]. This possible interpretation of the local mode peaks on the 580-nm ZPL phonon band was supported by the fact that H19 centers were observed simultaneously with the characteristic features in Raman spectra 3 and 4 (Fig. 4) in diamonds irradiated with neutrons [17, 39] or implanted 
with ions [39] at doses close to the graphitization level. Also, the band structure in the region of $\mathrm{H} 19$ center phonon repetitions allows other interpretations. The H19 center may have two ZPLs (580 and $598 \mathrm{~nm}$ ), which is characteristic of several other diamond optical centers, e.g., those formed by implantation of Xe (ZPL 794 and $811 \mathrm{~nm}$ [40]) or He ions (ZPL 536.5 and $560.5 \mathrm{~nm}[41])$.

Spectra in Figs. 1, 3, and 4 show also two narrow $(\Gamma \approx 3-4 \mathrm{~nm})$ PL maxima at 558 and $566 \mathrm{~nm}$ with intensities an order of magnitude less than that of the 580-nm ZPL. Previously, diamond PL maxima with similar spectral positions and $\Gamma$ values were observed [42] although their nature was not discussed. The intensity ratio of the maxima at 558 and $580 \mathrm{~nm}$ changed not only from sample to sample but also within individual crystallites (grains) in polycrystalline CVD diamonds irradiated by fast neutrons. The intensities of the ZPL maxima at 566 and $580 \mathrm{~nm}$ were correlated, which could indicate that the centers had similar natures.

On the nature of $H 19$ centers in diamond. The H19 optical center (ZPL $580 \mathrm{~nm}$ ) was observed only after hightemperature annealing and only in diamonds irradiated with high doses of light particles, e.g., hydrogen isotopes and neutrons. Irradiation with light particles causes less radiation damage due to secondary collisions of ejected $\mathrm{C}$ atoms, more point defects, and fewer collision cascades leading to the formation of disordered regions. It could be proposed that the H19 center formed during the reaction of simple point defects. The rate of such reactions depended linearly on the initial defect concentration. Therefore, $\mathrm{H} 19$ centers were observed only at high irradiation doses. High annealing temperatures are known to be required to form complex defects in diamond [10]. One method for annealing vacancies in diamond is to form multivacancy complexes starting with divacancies. Thermally activated vacancies can diffuse in diamond to form chains and loops of different lengths.

The formation of $\mathrm{H} 19$ centers was not affected by the content and speciation of $\mathrm{N}$ and Si impurities or the content of initial or implanted hydrogen because the center characteristics were independent of implanted hydrogen isotopes. Also, centers were observed in neutron-irradiated crystals and in natural diamond. The thermal and deformation softness of the H19 center indicated that it originated in a vacancy. This suggested that the H19 optical center was a complex intrinsic vacancy defect. The intensities of peaks at 335,1385 , and $1412 \mathrm{~cm}^{-1}$ increased simultaneously with the strengthening of the 580-nm band in PL and Raman spectra of diamonds irradiated by fast neutrons (Fig. 4). This could indicate that the $\mathrm{H} 19$ center contained a $\mathrm{C}=\mathrm{C}$ group.

Low-temperature piezospectroscopic measurements of PL spectra and ESR signal anisotropy of multivacancy centers should also be investigated to elucidate the details of the H19 optical center structure and symmetry [43].

Conclusions. PL spectra of natural and CVD diamonds irradiated by fast neutrons or implanted with hydrogen isotopes and annealed at high temperatures showed a ZPL at $580 \mathrm{~nm}$ and a structured phonon tail (H19 optical center). The strength and behavior of this band after annealing did not correlate with the concentration and speciation of $\mathrm{N}$ and $\mathrm{Si}$ in the studied diamonds or with the content of initial or implanted H/D. The activation energy for formation of the H19 center was $\geq 1.7 \mathrm{eV}$. The deformation and thermal softness of the $\mathrm{H} 19$ center were determined from the temperature dependence of PL spectra and the impact of local mechanical stresses on the shape of the peak with a ZPL at $580 \mathrm{~mm}$. A possible interpretation of the features on the $\mathrm{H} 19$ center phonon tail was proposed. The relationship of the H19 center and associated maxima at 558 and $566 \mathrm{~nm}$ was discussed. The results confirmed that the H19 optical center in radiation-damaged and high-temperature annealed diamonds was a complex intrinsic vacancy defect.

Acknowledgment. The work was financially supported by the Russian Science Foundation (Project 18-72-00076).

\section{REFERENCES}

1. A. Sipahigil, R. E. Evans, D. D. Sukachev, M. J. Burek, J. Borregaard, M. K. Bhaskar, C. T. Nguyen, J. L. Pacheco, H. A. Atikian, C. Meuwly, R. M. Camacho, F. Jelezko, E. Bielejec, H. Park, M. Loncar, and M. D. Lukin, Science, 354, No. 6314, 847-850 (2016).

2. S. Johnson, P. R. Dolan, and J. M. Smith, Prog. Quant. Electron., 55, 129-165 (2017).

3. E. Ekimov, M. V. Kondrin, V. S. Krivobok, A. A. Khomich, I. I. Vlasov, R. A. Khmelnitskiy, T. Iwasaki, and M. Hatano, Diamond Relat. Mater., 93, 75-83 (2019).

4. T. Luhmann, N. Raatz, R. John, M. Lesik, J. Rodiger, M. Portail, D. Wildanger, F. Kleissler, K. Nordlund, A. Zaitsev, J.-F. Roch, A. Tallaire, J. Meijer, and S. Pezzagna, J. Phys. D: Appl. Phys., 51, No. 48, 483002 (2018).

5. B. Dischler, Handbook of Spectral Lines in Diamond. Vol. 1. Tables and Interpretations, New York, Springer (2012), pp. 14-64. 
6. A. V. Khomich, R. A. Khmel'nitskii, N. A. Poklonskii, N. M. Lapchuk, A. A. Khomich, V. A. Dravin, O. N. Poklonskaya,

E. E. Ashkinazi, I. I. Vlasov, E. V. Zavedeev, and V. G. Ral'chenko, Zh. Prikl. Spektrosk., 79, No. 4, $615-624$ (2012) [A. V. Khomich, R. A. Khmelnitsky, N. A. Poklonski, N. M. Lapchuk, A. A. Khomich, V. A. Dravin, O. N. Poklonskaya, E. E. Ashkinazi, I. I. Vlasov, E. V. Zavedeev, and V. G. Ralchenko, J. Appl. Spectrosc., 79, No. 4, $600-609$ (2012)].

7. A. A. Khomich, V. G. Ral'chenko, A. V. Khomich, I. I. Vlasov, R. A. Khmel'nitskii, and A. E. Kar'kin, Izv. Vyssh. Uchebn. Zaved., Khim. Khim. Tekhnol., 56, No. 5, 27-31 (2013).

8. A. V. Khomich, A. E. Kar'kin, V. G. Ral'chenko, I. I. Vlasov, A. A. Khomich, N. M. Lapchuk, O. N. Poklonskaya, R. A. Khmel'nitskii, A. A. Averin, and A. A. Shiryaev, in: Proc. 9th Int. Confrence "Interaction of Radiation with Matter" [in Russian], September 20-22, 2011, Minsk, Izd. Tsentr BGU, Minsk (2011), pp. 180-182.

9. C. D. Clark, R. W. Ditchburn, and H. B. Dyer, Proc. R. Soc. London, Ser. A, 234, 363-381 (1956).

10. A. M. Zaitsev, Optical Properties of Diamond. A Data Handbook, Springer, Berlin (2001), p. 262.

11. A. A. Khomich, A. A. Averin, O. N. Poklonskaya, A. I. Kovalev, M. V. Kozlova, R. A. Khmel'nitskii, V. G. Ral'chenko, N. A. Poklonskii, and A. V. Khomich, in: Proc. 12th Int. Conf. "Interaction of Radiation with Matter" [in Russian], September 19-22, 2017, Minsk, Izd. Tsentr BGU, Minsk (2017), pp. 75-77.

12. J. Forneris, P. Traina, D. G. Monticone, G. Amato, L. Boarino, G. Brida, I. P. Degiovanni, E. Enrico, E. Moreva, V. Grilj, N. Skukan, M. Jaksic, M. Genovese, and P. Olivero, Sci. Rep., 5, 15901 (2015).

13. J. Forneris, S. D. Tchernij, A. Tengattini, E. Enrico, V. Grilj, N. Skukan, G. Amato, L. Boarino, M. Jaksic, and P. Olivero, Carbon, 113, 76-86 (2017).

14. K. Wang, J. Steeds, and Z. Li, Diamond Relat. Mater., 25, 29-33 (2012).

15. J. W. Steeds, Nucl. Instrum. Methods Phys. Res., Sect. B, 269, 1702-1706 (2011).

16. S. V. Nistor, M. Stefan, V. Ralchenko, A. Khomich, and D. Schoemaker, J. Appl. Phys., 87, 8741-8746 (2000).

17. A. V. Khomich, R. A. Khmel'nitskii, X. J. Hu, A. A. Khomich, A. F. Popovich, I. I. Vlasov, V. A. Dravin, Y. G. Chen, A. E. Kar'kin, and V. G. Ral'chenko, Zh. Prikl. Spektrosk., 80, No. 5, 719-726 (2013) [A. V. Khomich, R. A. Khmelnitskii, X. J. Hu, A. A. Khomich, A. F. Popovich, I. I. Vlasov, V. A. Dravin, Y. G. Chen, A. E. Karkin, and V. G. Ralchenko, J. Appl. Spectrosc., 80, 707-714 (2013)].

18. A. Bolshakov, V. Ralchenko, V. Sedov, A. Khomich, I. Vlasov, A. Khomich, N. Trofimov, V. Krivobok, S. Nikolaev, R. Khmelnitskii, and V. Saraykin, Phys. Status Solidi A, 212, 2525-2532 (2015).

19. A. V. Khomich, V. G. Ralchenko, A. V. Vlasov, R. A. Khmelnitskiy, I. I. Vlasov, and V. I. Konov, Diamond Relat. Mater., 10, 546-551 (2001).

20. A. A. Gippius, A. M. Zaitsev, and V. S. Vavilov, Fiz. Tekh. Poluprovodn., 16, 404-411 (1982) [A. A. Gippius, A. M. Zaitsev, and V. S. Vavilov, Sov. Phys. Semicond., 16, 256-261 (1982)].

21. A. A. Gippius, R. A. Khmelnitskiy, V. A. Dravin, and A. V. Khomich, Diamond Relat. Mater, 12, $538-541$ (2003).

22. K. Iakoubovskii, G. J. Adriaenssens, and M. Nesladek, J. Phys.: Condens. Matter, 12, 189-199 (2000).

23. R. U. A. Khan, P. M. Martineau, B. L. Cann, M. E. Newton, and D. J. Twitchen, J. Phys.: Condens. Matter, 21, 364214 (2009).

24. S. Onoda, K. Tatsumi, M. Haruyama, T. Teraji, J. Isoya, W. Kada, T. Ohshima, and O. Hanaizumi, Phys. Status Solidi A, 214, 1700160 (2017).

25. G. Davies and A. T. Collins, Diamond Relat. Mater., 2, 80-86 (1993).

26. E. Anastassakis, J. Appl. Phys., 86, 249-258 (1999).

27. I. I. Vlasov, V. G. Ralchenko, E. D. Obraztsova, A. A. Smolin, and V. I. Konov, Appl. Phys. Lett., 71, 1789-1791 (1997).

28. L. Nistor, V. Ralchenko, I. Vlasov, A. Khomich, R. Khmelnitskiy, P. Potapov, and J. Van Landuyt, Phys. Status Solidi A, 186, 207-214 (2001).

29. V. Ralchenko, L. Nistor, E. Pleuler, A. Khomich, I. Vlasov, and R. Khmelnitskii, Diamond Relat. Mater., 12, 1964-1970 (2003).

30. A. A. Khomich, A. N. Dzeraviaha, O. N. Poklonskaya, A. V. Khomich, R. A. Khmelnitsky, N. A. Poklonski, and V. G. Ralchenko, J. Phys.: Conf. Ser., 1135, 012019 (2018).

31. K. K. Rebane, Impurity Spectra of Solids: Elementary Theory of Vibrational Structure, Plenum Press, New York, London (1970), p. 45.

32. V. Hizhnyakov, H. Kaasik, and I. Sildos, Phys. Status Solidi B, 234, 644-653 (2002).

33. M. W. Doherty, V. M. Acosta, A. Jarmola, M. S. J. Barson, N. B. Manson, D. Budker, and L. C. L. Hollenberg, Phys. Rev. B: Condens. Matter Mater. Phys., 90, 041201(R) (2014). 
34. G. Davies, in: CVD Diamond for Electronic Devices and Sensors, R. S. Sussmann (Ed.), Wiley, Chichester (2009), pp. 3-28.

35. A. M. Zaitsev, Phys. Rev. B: Condens. Matter Mater. Phys., 61, 12909-12922 (2000).

36. W. Windl, P. Pavone, K. Karch, O. Schutt, D. Strauch, P. Giannozzi, and S. Baroni, Phys. Rev. B: Condens. Matter Mater. Phys., 48, 3164-3170 (1993).

37. P. Nemeth, L. A. J. Garvie, T. Aoki, N. Dubrovinskaia, L. Dubrovinsky, and P. R. Buseck, Nat. Commun., 5, 5447 (2014).

38. S. M. Pimenov, A. A. Khomich, I. I. Vlasov, E. V. Zavedeev, A. V. Khomich, B. Neuenschwander, B. Jaggi, and V. Romano, Appl. Phys. A: Mater. Sci. Process., 116, 545-554 (2014).

39. O. N. Poklonskaya, S. A. Vyrko, A. A. Khomich, A. A. Averin, A. V. Khomich, R. A. Khmel'nitskii, and N. A. Poklonskii, Zh. Prikl. Spektrosk., 81, No. 6, 879-887 (2014) [O. N. Poklonskaya, S. A. Vyrko, A. A. Khomich, A. A. Averin, A. V. Khomich, R. A. Khmelnitsky, and N. A. Poklonski, J. Appl. Spectrosc., 81, No. 6, 969-977 (2015)].

40. R. Sandstrom, L. Ke, A. Martin, Z. Wang, M. Kianinia, B. Green, W. Gao, and I. Aharonovich, Opt. Commun., 411, $182-186$ (2018).

41. V. D. Tkachev, A. M. Zaitsev, and V. V. Tkachev, Phys. Status Solidi B, 129, 129-133 (1985).

42. M. Wang, G. Shi, J. C. C. Yuan, W. Han, and Q. Bai, J. Spectrosc., 2018, 8153941 (2018).

43. Diamond (C), EPR spectra W1-W44, in: Impurities and Defects in Group IV Elements, IV-IV and III-V Compounds. Part a: Group IV Elements, O. Madelung, U. Rossler, and M. Schulz (Eds.), Springer, Berlin (2002), pp. 1-22. 